\title{
The online data pre-processing for CBM-TOF
}

\author{
C. Xiang ${ }^{* 1,2}$, N. Herrmann ${ }^{2}$, P.-A Loizeau ${ }^{2}$, I. Deppner ${ }^{2}$, S. Manz ${ }^{3}$, J. Frühauf ${ }^{4}$, and S. Linev ${ }^{4}$ \\ ${ }^{1}$ Key Laboratory of Quark and Lepton Physics (MOE) and Institute of Particle Physics, Central China Normal \\ University, China; ${ }^{2}$ Physikalisches Institut, Universität Heidelberg, Germany; ${ }^{3}$ Kirchhoff-Institut für Physik, \\ Heidelberg, Germany; ${ }^{4}$ GSI, Darmstadt, Germany
}

The Compressed Baryonic Matter (CBM) experiment will operate a free streaming data acquisition system. In order to optimize the data bandwidth and to achieve high performance, a data pre-processing is designed in the readout chain in ROC firmware [1] for the CBM-TOF detector. It consists of three modules, data pre-processing module, monitor module and control module [2]. The data preprocessing module (DPM) which is the main part has two functions, hit building and cluster building, which are designed in two steps. See Fig. 1.

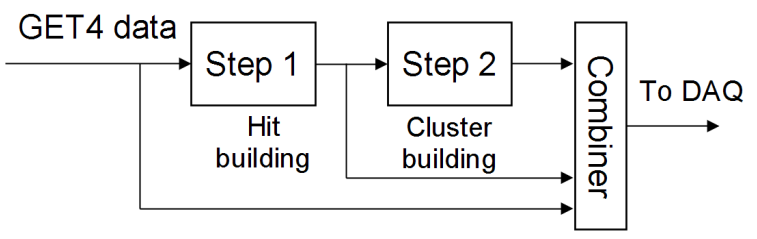

Figure 1: Structure of data pre-processing module

In the figure, the hit in the first step is built from rising edge and matched falling edge of GET4 data [3]. It has time information which is the rising edge time stamp and Time-Over-Threshold (TOT) information which is the time difference of rising edge and falling edge. The cluster in step 2 is a group of hit from 8 channels of two GET4 chips which connect to the two side of 4 neighbour Resistive Plate Chamber (RPC) strips. The time of the hits in the same cluster belongs to a certain time slice. All the data are combined together and sent to DAQ for cross checking.

Table 1: Data with/without DPM

\begin{tabular}{|l|c|c|c|}
\hline & RAW data & DPM data & \\
\hline Number of data & $2.28 \mathrm{E}+08$ & $1.39 \mathrm{E}+06$ & $99.4 \%$ \\
\hline Number of hits & 967749 & 967604 & $0.015 \%$ \\
\hline Number of events & 237856 & 237848 & $0.003 \%$ \\
\hline
\end{tabular}

The ROC firmware with 1st step was tested at COSY with a proton beam in November 2011 with MMRPC detector[4]. A possible reduction of the data volume by means of online pre-processing with 1st step DPM is demonstrated, see Tab. 1. In the table, the 2nd column Raw data is the result from offline analysis on GET4 data, and the 3rd column DPM data is the result of online analysis from the 1st step data pre-processing on GET4 data. The

\footnotetext{
*chxiang@physi.uni-heidelberg.de. Work supported by China Scholarship Council and BMBF 06HD9121I and EU/FP7 I3 Hadron Physics 2 .
}

last column show the result difference between offline analysis and online analysis. The final reduction of the output volume of the data depends on the hit rate, because most of the reduction is due to the rejection of the epoch data. In the case of hit rates in the order of $50 \mathrm{~Hz}$ per channel, as encountered during the test beam-time, about $99.4 \%$ reduction was achieved. As shown in table 1, during the test which run more than 49 minutes, the DPM lost only 145 hits $(0.015 \%)$, and only 8 events $(0.003 \%)$.

The ROC firmware in which cluster building was implemented was test at lab in July 2012. The input signal of these two channels were from a splitter with input connect to signal generator. The result is illustrated in table 2 . In principle, the number of Raw data from GET4 chip on these two channel should have same value. But in reality, they are different, see the 2 nd row of the table. After the 2nd step of data pre-processing, the number of two channels are the same. This shows a single hit in a time slice will be rejected as the single hit is meaningless from detector physical point of view.

Table 2: Number of obtained hits from different data preprocessing level

\begin{tabular}{|c|c|c|}
\hline & Chn. 1 & Chn. 6 \\
\hline Raw data & 2507905 & 2507991 \\
\hline 1st step DPM data & 2507243 & 2507217 \\
\hline Hit lost & $0.0264 \%$ & $0.0309 \%$ \\
\hline 2nd step DPM data & 2506236 & 2506236 \\
\hline Hit lost & $0.07 \%$ & $0.07 \%$ \\
\hline
\end{tabular}

These two test shows the data pre-processing module was fully functional and demonstrated the potential of implementing an on-line inspection of the data. Further features, like threshold self-adjusting can also be done in the readout chain.

\section{References}

[1] Manz, S., et al. "An universal read-out controller." Journal of Instrumentation 5.11 (2010): C11017.

[2] C.Xiang, FPGA based control and monitor for CBM-TOF readout, CBM Progress Report 2011

[3] H. Flemming and H. Deppe, "The GSI event-driven TDC with 4 channels GET4",IEEE NSS, Orlando, FL, (2009) 1082-3654

[4] Deppner, I., et al. "A glass type Multistrip-MRPC prototype for the low rate region of the CBM Time-of-Flight wall." Journal of Instrumentation 7.10 (2012): P10008. 